\title{
Homoclinic Solutions of Singular Nonautonomous Second-Order Differential Equations
}

\author{
Irena Rachůnková and Jan Tomeček \\ Department of Mathematical Analysis and Applications of Mathematics, Faculty of Science, \\ Palacký University, 17 listopadu 12, 77146 Olomouc, Czech Republic \\ Correspondence should be addressed to Irena Rachůnková, rachunko@inf.upol.cz \\ Received 27 April 2009; Revised 1 September 2009; Accepted 15 September 2009 \\ Recommended by Donal O'Regan
}

This paper investigates the singular differential equation $\left(p(t) u^{\prime}\right)^{\prime}=p(t) f(u)$, having a singularity at $t=0$. The existence of a strictly increasing solution (a homoclinic solution) satisfying $u^{\prime}(0)=0$, $u(\infty)=L>0$ is proved provided that $f$ has two zeros and a linear behaviour near $-\infty$.

Copyright ( 2009 I. Rachůnková and J. Tomeček. This is an open access article distributed under the Creative Commons Attribution License, which permits unrestricted use, distribution, and reproduction in any medium, provided the original work is properly cited.

\section{Introduction}

Having a positive parameter $L$, we consider the problem

$$
\begin{gathered}
\left(p(t) u^{\prime}\right)^{\prime}=p(t) f(u), \\
u^{\prime}(0)=0, \quad u(\infty)=L,
\end{gathered}
$$

under the following basic assumptions for $f$ and $p$

$$
\begin{gathered}
f \in \operatorname{Lip}_{\text {loc }}((-\infty, L]), \quad f(0)=f(L)=0, \\
f(x)<0 \quad \text { for } x \in(0, L),
\end{gathered}
$$

there exists $\bar{B}<0$ such that $f(x)>0$ for $x \in[\bar{B}, 0)$,

$$
\begin{gathered}
F(\bar{B})=F(L), \quad \text { where } F(x)=-\int_{0}^{x} f(z) \mathrm{d} z, \\
p \in C([0, \infty)) \cap C^{1}((0, \infty)), \quad p(0)=0, \\
p^{\prime}(t)>0, t \in(0, \infty), \quad \lim _{t \rightarrow \infty} \frac{p^{\prime}(t)}{p(t)}=0 .
\end{gathered}
$$


Then problem (1.1), (1.2) generalizes some models arising in hydrodynamics or in the nonlinear field theory (see [1-5]). However (1.1) is singular at $t=0$ because $p(0)=0$.

Definition 1.1. If $c>0$, then a solution of $(1.1)$ on $[0, c]$ is a function $u \in C^{1}([0, c]) \cap C^{2}((0, c])$ satisfying $(1.1)$ on $(0, c]$. If $u$ is a solution of $(1.1)$ on $[0, c]$ for each $c>0$, then $u$ is a solution of $(1.1)$ on $[0, \infty)$.

Definition 1.2. Let $u$ be a solution of $(1.1)$ on $[0, \infty)$. If $u$ moreover fulfils conditions (1.2), it is called a solution of problem (1.1), (1.2).

Clearly, the constant function $u(t) \equiv L$ is a solution of problem (1.1), (1.2). An important question is the existence of a strictly increasing solution of (1.1), (1.2) because if such a solution exists, many important physical properties of corresponding models can be obtained. Note that if we extend the function $p(t)$ in (1.1) from the half-line onto $\mathbb{R}$ (as an even function), then any solution of (1.1), (1.2) has the same limit $L$ as $t \rightarrow-\infty$ and $t \rightarrow \infty$. Therefore we will use the following definition.

Definition 1.3. A strictly increasing solution of problem (1.1), (1.2) is called a homoclinic solution.

Numerical investigation of problem (1.1), (1.2), where $p(t)=t^{2}$ and $f(u)=4 \lambda^{2}(u+$ 1) $u(u-L), \lambda>0$, can be found in $[1,4-6]$. Problem (1.1), (1.2) can be also transformed onto a problem about the existence of a positive solution on the half-line. For $p(t)=t^{k}, k \in \mathbb{N}$ and for $p(t)=t^{k}, k \in(1, \infty)$, such transformed problem was solved by variational methods in $[7,8]$, respectively. Some additional assumptions imposed on $f$ were needed there. Related problems were solved, for example, in $[9,10]$.

Here, we deal directly with problem (1.1), (1.2) and continue our earlier considerations of papers $[11,12]$, where we looked for additional conditions which together with (1.3)-(1.8) would guarantee the existence of a homoclinic solution.

Let us characterize some results reached in $[11,12]$ in more details. Both these papers assume (1.3)-(1.8). In [11] we study the case that $f$ has at least three zeros $L_{0}<0<L$. More precisely, the conditions,

$$
\begin{gathered}
f\left(L_{0}\right)=0 \text {, there exists } \delta>0 \text { such that } f \in C^{1}((-\delta, 0)), \lim _{x \rightarrow 0-} f^{\prime}(x)<0, \\
p \in C^{2}((0, \infty)), \quad \lim _{t \rightarrow \infty} \frac{p^{\prime \prime}(t)}{p(t)}=0,
\end{gathered}
$$

are moreover assumed. Then there exist $c>0, B \in\left(L_{0}, 0\right)$, and a solution $u$ of $(1.1)$ on $[0, c]$ such that

$$
\begin{gathered}
u(0)=B, \quad u^{\prime}(0)=0, \\
u^{\prime}(t)>0 \text { for } t \in(0, c], \quad u(c)=L .
\end{gathered}
$$

We call such solution an escape solution. The main result of [11] is that (under (1.3)-(1.8), (1.9)) the set of solutions of (1.1), (1.10) for $B \in\left(L_{0}, 0\right)$ consists of escape solutions and of oscillatory solutions (having values in $\left(L_{0}, L\right)$ ) and of at least one homoclinic solution. 
In [12] we omit assumptions (1.9) and prove that assumptions (1.3)-(1.8) are sufficient for the existence of an escape solution and also for the existence of a homoclinic solution provided the $p$ fulfils

$$
\int_{0}^{1} \frac{\mathrm{d} s}{p(s)}<\infty
$$

If (1.12) is not valid, then the existence of both an escape solution and a homoclinic solution is proved in [12], provided that $f$ satisfies moreover

$$
\begin{gathered}
f(x)>0 \quad \text { for } x<0, \\
\lim _{x \rightarrow-\infty} \frac{|x|}{f(x)}=\infty .
\end{gathered}
$$

Assumption (1.13) characterizes the case that $f$ has just two zeros 0 and $L$ in the interval $(-\infty, L]$. Further, we see that if (1.14) holds, then $f$ is either bounded on $(-\infty, L]$ or $f$ is unbounded earlier and has a sublinear behaviour near $-\infty$.

This paper also deals with the case that $f$ satisfies (1.13) and is unbounded above on $(-\infty, L]$. In contrast to [12], here we prove the existence of a homoclinic solution for $f$ having a linear behaviour near $-\infty$. The proof is based on a full description of the set of all solutions of problem (1.1), (1.10) for $B<0$ and on the existence of an escape solutions in this set.

Finally, we want to mention the paper [13], where the problem

$$
\begin{gathered}
\frac{1}{p(t)}\left(p(t) u^{\prime}(t)\right)^{\prime}=f\left(t, u(t), p(t) u^{\prime}(t)\right), \\
u(0)=\rho_{0} \in(-1,0), \quad \lim _{t \rightarrow \infty} u(t)=\xi \in(0,1), \\
\lim _{t \rightarrow \infty} p(t) u^{\prime}(t)=0
\end{gathered}
$$

is investigated under the assumptions that $f$ is continuous, it has three distinct zeros and satisfies the sign conditions similar to those in [11, (3.4)]. In [13], an approach quite different from $[11,12]$ is used. In particular, by means of properties of the associated vector field $\left(u(t), p(t) u^{\prime}(t)\right)$ together with the Kneser's property of the cross sections of the solutions' funnel, the authors provide conditions which guarantee the existence of a strictly increasing solution of (1.15). The authors apply this general result to problem

$$
\begin{aligned}
& \frac{1}{t^{n-1}}\left(t^{n-1} u^{\prime}\right)^{\prime}=4 \lambda^{2}(u+1) u(u-\xi), \\
& \lim _{t \rightarrow 0+} t^{n-1} u^{\prime}(t)=0, \quad \lim _{t \rightarrow \infty} u(t)=\xi,
\end{aligned}
$$

and get a strictly increasing solution of (1.16) for a sufficiently small $\xi$. This corresponds to the results of $[11]$, where $\xi \in(0,1)$ may be arbitrary. 


\section{Initial Value Problem}

In this section, under the assumptions (1.3)-(1.8) and (1.13) we prove some basic properties of solutions of the initial value problem (1.1), (1.10), where $B<0$.

Lemma 2.1. For each $B<0$ there exists a maximal $c^{*} \in(0, \infty]$ such that problem (1.1), (1.10) has a unique solution $u$ on $\left[0, c^{*}\right)$ and

$$
u(t) \geq B \quad \text { for } t \in\left[0, c^{*}\right) .
$$

Further, for each $b \in\left(0, c^{*}\right)$, there exists $M_{b}>0$ such that

$$
|u(t)|+\left|u^{\prime}(t)\right| \leq M_{b}, \quad t \in[0, b], \quad \int_{0}^{b} \frac{p^{\prime}(s)}{p(s)}\left|u^{\prime}(s)\right| \mathrm{d} s \leq M_{b} .
$$

Proof. Let $u$ be a solution of problem $(1.1),(1.10)$ on $[0, c) \subset[0, \infty)$. By $(1.1)$, we have

$$
u^{\prime \prime}(t)+\frac{p^{\prime}(t)}{p(t)} u^{\prime}(t)-f(u(t))=0 \quad \text { for } t \in(0, c)
$$

and multiplying by $u^{\prime}$ and integrating between 0 and $t$, we get

$$
\frac{u^{\prime 2}(t)}{2}+\int_{0}^{t} \frac{p^{\prime}(s)}{p(s)} u^{\prime 2}(s) \mathrm{d} s+F(u(t))=F(B), \quad t \in(0, c)
$$

Let $u\left(t_{1}\right)<B$ for some $t_{1} \in(0, c)$. Then (2.4) yields $F\left(u\left(t_{1}\right)\right) \leq F(B)$, which is not possible, because $F$ is decreasing on $(-\infty, 0)$. Therefore $u(t) \geq B$ for $t \in[0, c)$.

Let $\eta>0$. Consider the Banach space $C([0, \eta])$ (with the maximum norm) and an operator $\mathcal{F}: C([0, \eta]) \rightarrow C([0, \eta])$ defined by

$$
(\mp u)(t)=B+\int_{0}^{t} \frac{1}{p(s)} \int_{0}^{s} p(\tau) f(u(\tau)) \mathrm{d} \tau \mathrm{d} s
$$

A function $u$ is a solution of problem $(1.1),(1.2)$ on $[0, \eta]$ if and only if it is a fixed point of the operator $\mathbb{F}$. Using the Lipschitz property of $f$ we can prove that the operator is contractive for each sufficiently small $\eta$ and from the Banach Fixed Point Theorem we conclude that there exists exactly one solution of problem $(1.1),(1.2)$ on $[0, \eta]$. This solution $u$ has the form

$$
u(t)=B+\int_{0}^{t} \frac{1}{p(s)} \int_{0}^{s} p(\tau) f(u(\tau)) \mathrm{d} \tau \mathrm{d} s
$$

for $t \in[0, \eta]$. Hence, $u$ can be extended onto each interval $[0, b]$ where $u$ is bounded. So, we can put $c^{*}=\sup \{b>0: u$ is bounded on $[0, b]\}$. 
Let $b \in\left(0, c^{*}\right)$. Then there exists $\widetilde{M} \in(0, \infty)$ such that $|f(u(t))| \leq \widetilde{M}$ for $t \in[0, b]$. So, (2.6) yields

$$
\left|u^{\prime}(t)\right| \leq \widetilde{M} \frac{1}{p(t)} \int_{0}^{t} p(s) \mathrm{d} s, \quad t \in(0, b]
$$

Put

$$
\varphi(t)=\frac{1}{p(t)} \int_{0}^{t} p(s) \mathrm{d} s, \quad \psi(t)=\int_{t}^{b} \frac{p^{\prime}(s)}{p^{2}(s)} \int_{0}^{s} p(\tau) \mathrm{d} \tau \mathrm{d} s, \quad t \in(0, b]
$$

Then

$$
0<\varphi(t) \leq t \quad \text { for } t \in(0, b]
$$

and, by "per partes" integration we derive $\lim _{t \rightarrow 0+} \psi(t)=b-\varphi(b)$. Multiplying (2.7) by $p^{\prime}(t) / p(t)$ and integrating it over $(0, b)$, we get

$$
\int_{0}^{b} \frac{p^{\prime}(t)}{p(t)}\left|u^{\prime}(t)\right| \mathrm{d} t \leq \widetilde{M} \int_{0}^{b} \frac{p^{\prime}(t)}{p^{2}(t)} \int_{0}^{t} p(s) \mathrm{d} s \mathrm{~d} t=\widetilde{M}(b-\varphi(b)) .
$$

Estimates (2.2) follow from (2.7)-(2.10) for

$$
M_{b}=\widetilde{M} b+|B|+\widetilde{M} b^{2} .
$$

Remark 2.2. The proof of Lemma 2.1 yields that if $c^{*}<\infty$, then $\lim _{t \rightarrow c *} u(t)=\infty$.

Let us put

$$
\tilde{f}(x)= \begin{cases}0 & \text { for } x>L \\ f(x) & \text { for } x \leq L\end{cases}
$$

and consider an auxiliary equation

$$
\left(p(t) u^{\prime}\right)^{\prime}=p(t) \tilde{f}(u)
$$

Similarly as in the proof of Lemma 2.1 we deduce that problem (2.13), (1.10) has a unique solution on $[0, \infty)$. Moreover the following lemma is true.

Lemma 2.3 ([12]). For each $B_{0}<0, b>0$ and each $\epsilon>0$, there exists $\delta>0$ such that for any $B_{1}$, $B_{2} \in\left[B_{0}, 0\right)$

$$
\left|B_{1}-B_{2}\right|<\delta \Longrightarrow\left|u_{1}(t)-u_{2}(t)\right|+\left|u_{1}^{\prime}(t)-u_{2}^{\prime}(t)\right|<\epsilon, \quad t \in[0, b]
$$

Here $u_{i}$ is a solution of problem (2.13), (1.10) with $B=B_{i}, i=1,2$. 
Proof. Choose $B_{0}<0, b>0, \epsilon>0$. Let $K>0$ be the Lipschitz constant for $f$ on $\left[B_{0}, L\right]$. By (2.6) for $f=\tilde{f}, B=B_{i}, u=u_{i}, i=1,2$,

$$
\begin{aligned}
\left|u_{1}(t)-u_{2}(t)\right| & \leq\left|B_{1}-B_{2}\right|+\int_{0}^{t} \frac{1}{p(s)} \int_{0}^{s} p(\tau)\left|\tilde{f}\left(u_{1}(\tau)\right)-\tilde{f}\left(u_{2}(\tau)\right)\right| \mathrm{d} \tau \mathrm{d} s \\
& \leq\left|B_{1}-B_{2}\right|+K t \int_{0}^{t}\left|u_{1}(\tau)-u_{2}(\tau)\right| \mathrm{d} \tau \\
& \leq\left|B_{1}-B_{2}\right|+K b \int_{0}^{t}\left|u_{1}(\tau)-u_{2}(\tau)\right| \mathrm{d} \tau, \quad t \in[0, b]
\end{aligned}
$$

From the Gronwall inequality, we get

$$
\left|u_{1}(t)-u_{2}(t)\right| \leq\left|B_{1}-B_{2}\right| e^{K b^{2}}, \quad t \in[0, b] .
$$

Similarly, by (2.6), (2.9), and (2.16),

$$
\begin{aligned}
\left|u_{1}^{\prime}(t)-u_{2}^{\prime}(t)\right| & \leq \frac{1}{p(t)} \int_{0}^{t} p(s)\left|\tilde{f}\left(u_{1}(s)\right)-\tilde{f}\left(u_{2}(s)\right)\right| \mathrm{d} s \\
& \leq K \frac{1}{p(t)} \int_{0}^{t} p(s)\left|u_{1}(s)-u_{2}(s)\right| \mathrm{d} s \\
& \leq K b\left|B_{1}-B_{2}\right| e^{K b^{2}}, \quad t \in[0, b] .
\end{aligned}
$$

If we choose $\delta>0$ such that

$$
\delta<\frac{\epsilon}{(1+K b) e^{K b^{2}}}
$$

we get (2.14).

Remark 2.4. Choose $a \geq 0$ and $C \leq L$, and consider the initial conditions

$$
u(a)=C, \quad u^{\prime}(a)=0
$$

Arguing as in the proof of Lemma 2.1, we get that problem (2.13), (2.19) has a unique solution on $[a, \infty)$. In particular, for $C=0$ and $C=L$, the unique solution of problem (2.13), (2.19) (and also of problem (1.1), (2.19)) is $u \equiv 0$ and $u \equiv L$, respectively.

Lemma 2.5. Let $u$ be a solution of problem (1.1), (1.10). Assume that there exists $a \geq 0$ such that

$$
u(t)<0 \quad \text { for } t \geq a, \quad u^{\prime}(a)=0
$$


Then $u^{\prime}(t)>0$ for $t>a$ and

$$
\lim _{t \rightarrow \infty} u(t)=0, \quad \lim _{t \rightarrow \infty} u^{\prime}(t)=0 .
$$

Proof. By (1.13) and (2.20), $f(u(t))>0$ on $[a, \infty)$ and thus $p(t) u^{\prime}(t)$ and $u^{\prime}(t)$ are positive on $(a, \infty)$. Consequently, there exists $\lim _{t \rightarrow \infty} u(t)=B_{1} \in(u(a), 0]$. Further, by (1.1),

$$
u^{\prime \prime}(t)+\frac{p^{\prime}(t)}{p(t)} u^{\prime}(t)=f(u(t)), \quad t>0,
$$

and, by multiplication and integration over $[a, t]$,

$$
\frac{u^{\prime 2}(t)}{2}+\int_{a}^{t} \frac{p^{\prime}(s)}{p(s)} u^{\prime 2}(s) \mathrm{d} s=F(u(a))-F(u(t)), \quad t>a .
$$

Therefore,

$$
0 \leq \lim _{t \rightarrow \infty} \int_{a}^{t} \frac{p^{\prime}(s)}{p(s)} u^{\prime 2}(s) \mathrm{d} s \leq F(u(a))-F\left(B_{1}\right)<\infty,
$$

and hence $\lim _{t \rightarrow \infty} u^{\prime 2}(t)$ exists. Since $u$ is bounded on $[0, \infty)$, we get

$$
\lim _{t \rightarrow \infty} u^{\prime 2}(t)=\lim _{t \rightarrow \infty} u^{\prime}(t)=0
$$

By (1.3), (1.8), and (2.22), $\lim _{t \rightarrow \infty} u^{\prime \prime}(t)$ exists and, since $u^{\prime}$ is bounded on $[0, \infty)$, we get $\lim _{t \rightarrow \infty} u^{\prime \prime}(t)=0$. Hence, letting $t \rightarrow \infty$ in (2.22), we obtain $f\left(B_{1}\right)=0$. Therefore, $B_{1}=0$ and (2.21) is proved.

Lemma 2.6. Let $u$ be a solution of problem (1.1), (1.10). Assume that there exist $a_{1}>0$ and $A_{1} \in$ $(0, L)$ such that

$$
u(t)>0 \quad \forall t>a_{1}, \quad u\left(a_{1}\right)=A_{1}, \quad u^{\prime}\left(a_{1}\right)=0 .
$$

Then $u^{\prime}(t)<0$ for all $t>a_{1}$ and (2.21) holds.

Proof. Since $u$ fulfils (2.26), we can find a maximal $b>a_{1}$ such that $0<u(t)<L$ for $t \in\left[a_{1}, b\right)$ and consequently $f(u(t))=\tilde{f}(u(t))$ for $t \in\left[a_{1}, b\right)$. By (4.23) and (2.26), $f(u(t))<0$ on $\left[a_{1}, b\right)$ and thus $p(t) u^{\prime}(t)$ and $u^{\prime}(t)$ are negative on $\left(a_{1}, b\right)$. So, $u$ is positive and decreasing on $\left[a_{1}, b\right)$ which yields $b=\infty$ (otherwise, we get $u(b)=0$, contrary to (2.26)). Consequently there exists $\lim _{t \rightarrow \infty} u(t)=L_{1} \in\left[0, A_{1}\right)$. By multiplication and integration $(2.22)$ over $\left[a_{1}, t\right]$, we obtain

$$
\frac{u^{\prime 2}(t)}{2}+\int_{a_{1}}^{t} \frac{p^{\prime}(s)}{p(s)} u^{\prime 2}(s) \mathrm{d} s=F\left(A_{1}\right)-F(u(t)), \quad t>a_{1} .
$$


By similar argument as in the proof of Lemma 2.5 we get that $\lim _{t \rightarrow \infty} \mathcal{u}^{\prime}(t)=0$ and $L_{1}=0$. Therefore (2.21) is proved.

\section{Damped Solutions}

In this section, under assumptions (1.3)-(1.8) and (1.13) we describe a set of all damped solutions which are defined in the following way.

Definition 3.1. A solution of problem (1.1), (1.10) (or of problem (2.13), (1.10)) on $[0, \infty)$ is called damped if

$$
\sup \{u(t): t \in[0, \infty)\}<L
$$

Remark 3.2. We see, by (2.12), that $u$ is a damped solution of problem (1.1), (1.10) if and only if $u$ is a damped solution of problem (2.13), (1.10). Therefore, we can borrow the arguments of [12] in the proofs of this section.

Theorem 3.3. If $u$ is a damped solution of problem (1.1), (1.10), then $u$ has a finite number of isolated zeros and satisfies (2.21); or $u$ is oscillatory (it has an unbounded set of isolated zeros).

Proof. Let $u$ be a damped solution of problem (1.1), (1.10). By Remark 2.2, we have $c^{*}=\infty$ in Lemma 2.1 and hence

$$
u(t) \geq B \quad \text { for } t \in[0, \infty)
$$

Step 1. If $u$ has no zero in $(0, \infty)$, then $u(t)<0$ for $t \geq 0$ and, by Lemma $2.5, u$ fulfils (2.21).

Step 2. Assume that $\theta>0$ is the first zero of $u$ on $(0, \infty)$. Then, due to Remark 2.4, $u^{\prime}(\theta)>0$. Let $u(t)>0$ for $t \in(\theta, \infty)$. By virtue of $(1.4), f(u(t))<0$ for $t \in(\theta, \infty)$ and thus $p(t) u^{\prime}(t)$ is decreasing. Let $u^{\prime}$ be positive on $(\theta, \infty)$. Then $u^{\prime}$ is also decreasing, $u$ is increasing and $\lim _{t \rightarrow \infty} u(t)=\bar{L} \in(0, L)$, due to (3.1). Consequently, $\lim _{t \rightarrow \infty} u^{\prime}(t)=0$. Letting $t \rightarrow \infty$ in (2.22), we get $\lim _{t \rightarrow \infty} u^{\prime \prime}(t)=f(\bar{L})<0$, which is impossible because $u^{\prime}$ is bounded below. Therefore there are $a_{1}>\theta$ and $A_{1} \in(0, L)$ satisfying (2.26) and, by Lemma 2.6, either $u$ fulfils (2.21) or $u$ has the second zero $\theta_{1}>a_{1}$ with $u^{\prime}\left(\theta_{1}\right)<0$. So $u$ is positive on $\left(\theta, \theta_{1}\right)$ and has just one local maximum $A_{1}=u\left(a_{1}\right)$ in $\left(\theta, \theta_{1}\right)$. Moreover, putting $a=0$ and $t=a_{1}$ in (2.23), we have

$$
0<\int_{0}^{a_{1}} \frac{p^{\prime}(s)}{p(s)} u^{\prime 2}(s) \mathrm{d} s=F(B)-F\left(A_{1}\right)
$$

and hence

$$
F\left(A_{1}\right)<F(B) .
$$


Step 3. Let $u$ have no other zeros. Then $u(t)<0$ for $t \in\left(\theta_{1}, \infty\right)$. Assume that $u^{\prime}$ is negative on $\left[\theta_{1}, \infty\right)$. Then, due to $(2.1), \lim _{t \rightarrow \infty} u(t)=\bar{L} \in[B, 0)$. Putting $a=a_{1}$ in (2.23) and letting $t \rightarrow \infty$, we obtain

$$
0<\lim _{t \rightarrow \infty}\left[\frac{u^{\prime 2}(t)}{2}+\int_{a_{1}}^{t} \frac{p^{\prime}(s)}{p(s)} u^{\prime 2}(s) \mathrm{d} s\right]=F\left(A_{1}\right)-F(\bar{L})
$$

Therefore, $\lim _{t \rightarrow \infty} u^{\prime 2}(t)$ exists and, since $u$ is bounded, we deduce that

$$
\lim _{t \rightarrow \infty} u^{\prime}(t)=0
$$

Letting $t \rightarrow \infty$ in (2.22), we get $\lim _{t \rightarrow \infty} u^{\prime \prime}(t)=f(\bar{L})>0$, which contradicts the fact that $u^{\prime}$ is bounded above. Therefore, $u^{\prime}$ cannot be negative on the whole interval $\left[\theta_{1}, \infty\right)$ and there exists $b_{1}>\theta_{1}$ such that $u^{\prime}\left(b_{1}\right)=0$. Moreover, according to $(3.2), u\left(b_{1}\right) \in[B, 0)$.

Then, Lemma 2.5 yields that $u$ fulfils (2.21). Since $u^{\prime}$ is positive on $\left(b_{1}, \infty\right), u$ has just one minimum $B_{1}=u\left(b_{1}\right)$ on $\left(\theta_{1}, \infty\right)$. Moreover, putting $a=a_{1}$ and $t=b_{1}$ in (2.23), we have

$$
0<\int_{a_{1}}^{b_{1}} \frac{p^{\prime}(s)}{p(s)} u^{\prime 2}(s) \mathrm{d} s=F\left(A_{1}\right)-F\left(B_{1}\right),
$$

which together with (3.4) yields

$$
F\left(B_{1}\right)<F\left(A_{1}\right)<F(B)
$$

Step 4. Assume that $u$ has its third zero $\theta_{2}>\theta_{1}$. Then we prove as in Step 2 that $u$ has just one negative minimum $B_{1}=u\left(b_{1}\right)$ in $\left(\theta_{1}, \theta_{2}\right)$ and (3.8) is valid. Further, as in Step 2, we deduce that either $u$ fulfils (2.21) or $u$ has the fourth zero $\theta_{3}>\theta_{2}, u$ is positive on $\left(\theta_{2}, \theta_{3}\right)$ with just one local maximum $A_{2}=u\left(a_{2}\right)<L$ on $\left(\theta_{2}, \theta_{3}\right)$, and $F\left(A_{2}\right)<F\left(B_{1}\right)$. This together with (3.8) yields

$$
F\left(A_{2}\right)<F\left(B_{1}\right)<F\left(A_{1}\right)<F(B) .
$$

If $u$ has no other zeros, we deduce as in Step 3 that $u$ has just one negative minimum $B_{2}=$ $u\left(b_{2}\right)$ in $\left(\theta_{3}, \infty\right), F\left(B_{2}\right)<F\left(A_{2}\right)$ and $u$ fulfils $(2.21)$.

Step 5. If $u$ has other zeros, we use the previous arguments and get that either $u$ has a finite number of zeros and then fulfils (2.21) or $u$ is oscillatory.

Remark 3.4. According to the proof of Theorem 3.3, we see that if $u$ is oscillatory, it has just one positive local maximum between the first and the second zero, then just one negative local minimum between the second and the third zero, and so on. By (3.8), (3.9), (1.4)-(1.6) and (1.13), these maxima are decreasing (minima are increasing) for $t$ increasing. 
Lemma 3.5. A solution u of problem (1.1), (1.10) fulfils the condition

$$
\sup \{u(t): t \in[0, \infty)\}=L
$$

if and only if $u$ fulfils the condition

$$
\lim _{t \rightarrow \infty} u(t)=L, \quad u^{\prime}(t)>0 \quad \text { for } t \in(0, \infty)
$$

Proof. Assume that $u$ fulfils (3.10). Then there exists $\theta \in(0, \infty)$ such that $u(\theta)=0, u^{\prime}(t)>0$ for $t \in(0, \theta]$. Otherwise $\sup \{u(t): t \in[0, \infty)\}=0$, due to Lemma 2.5. Let $a_{1} \in(\theta, \infty)$ be such that $u^{\prime}(t)>0$ on $\left(\theta, a_{1}\right), u^{\prime}\left(a_{1}\right)=0$. By Remark 2.4 and (3.10), $u\left(a_{1}\right) \in(0, L)$. Integrating (1.1) over $\left(a_{1}, t\right)$, we get

$$
u^{\prime}(t)=\frac{1}{p(t)} \int_{a_{1}}^{t} p(s) f(u(s)) \mathrm{d} s, \quad \forall t>a_{1}
$$

Due to (1.4), we see that $u$ is strictly decreasing for $t>a_{1}$ as long as $u(t) \in(0, L)$. Thus, there are two possibilities. If $u(t)>0$ for all $t>a_{1}$, then from Lemma 2.6 we get (2.21), which contradicts (3.10). If there exists $\theta_{1}>a_{1}$ such that $u\left(\theta_{1}\right)=0$, then in view Remark 2.4 we have $u^{\prime}\left(\theta_{1}\right)<0$. Using the arguments of Steps 3-5 of the proof of Theorem 3.3, we get that $u$ is damped, contrary to (3.10). Therefore, such $a_{1}$ cannot exist and $u^{\prime}>0$ on $(0, \infty)$. Consequently, $\lim _{t \rightarrow \infty} u(t)=L$. So, $u$ fulfils (3.11). The inverse implication is evident.

Remark 3.6. According to Definition 1.3 and Lemma 3.5, $u$ is a homoclinic solution of problem (1.1), (1.10) if and only if $u$ is a homoclinic solution of problem (2.13), (1.10).

Theorem 3.7 (on damped solutions). Let $\bar{B}$ satisfy (1.5) and (1.6). Assume that $u$ is a solution of problem (1.1), (1.10) with $B \in[\bar{B}, 0)$. Then $u$ is damped.

Proof. Let $u$ be a solution of (1.1), (1.10) with $B \in[\bar{B}, 0)$. Then, by (1.4)-(1.6),

$$
F(B) \leq F(L)
$$

Assume on the contrary that $u$ is not damped. Then $u$ is defined on the interval $[0, \infty)$ and $\sup \{u(t): t \in[0, \infty)\}=L$ or there exists $b \in(0, \infty)$ such that $u(b)=L, u^{\prime}(b)>0$, and $u(t)<L$ for $t \in[0, b)$. If the latter possibility occurs, (2.22) and (3.13) give by integration

$$
0<\frac{u^{\prime 2}(b)}{2}+\int_{0}^{b} \frac{p^{\prime}(s)}{p(s)} u^{\prime 2}(s) \mathrm{d} s=F(B)-F(L) \leq 0,
$$

a contradiction. If $\sup \{u(t): t \in[0, \infty)\}=L$, then, by Lemma 3.5, $u$ fulfils (3.11). So $u$ has a unique zero $\theta>0$. Integrating (2.22) over $[0, \theta]$, we get

$$
\frac{u^{\prime 2}(\theta)}{2}+\int_{0}^{\theta} \frac{p^{\prime}(s)}{p(s)} u^{\prime 2}(s) \mathrm{d} s=F(B)
$$


and so

$$
u^{\prime 2}(\theta)<2 F(B)
$$

Integrating (2.22) over $[\theta, t]$, we obtain for $t>\theta$

$$
\frac{u^{\prime 2}(t)}{2}-\frac{u^{\prime 2}(\theta)}{2}+\int_{\theta}^{t} \frac{p^{\prime}(s)}{p(s)} u^{\prime 2}(s) \mathrm{d} s=F(u(\theta))-F(u(t))=-F(u(t)) .
$$

Therefore, $u^{\prime 2}(\theta)>2 F(u(t))$ on $(\theta, \infty)$, and letting $t \rightarrow \infty$, we get $u^{\prime 2}(\theta) \geq 2 F(L)$. This together with (3.16) contradicts (3.13). We have proved that $u$ is damped.

Theorem 3.8. Let $\boldsymbol{M}_{d}$ be the set of all $B<0$ such that corresponding solutions of problem (1.1), (1.10) are damped. Then $\boldsymbol{M}_{d}$ is open in $(-\infty, 0)$.

Proof. Let $B_{0} \in \mathcal{M}_{d}$ and $u_{0}$ be a solution of (1.1), (1.10) with $B=B_{0}$. So, $u_{0}$ is damped and $u_{0}$ is also a solution of (2.13).

(a) Let $u_{0}$ be oscillatory. Then its first local maximum belongs to $(0, L)$. Lemma 2.3 guarantees that if $B$ is sufficiently close to $B_{0}$, the corresponding solution $u$ of $(2.13),(1.10)$ has also its first local maximum in $(0, L)$. This means that there exist $a_{1}>0$ and $A_{1} \in(0, L)$ such that $u$ satisfies (2.26). Now, we can continue as in the proof of Theorem 3.3 using the arguments of Steps $2-5$ and Remark 3.2 to get that $u$ is damped.

(b) Let $u_{0}$ have at most a finite number of zeros. Then, by Theorem 3.3, $u_{0}$ fulfils (2.21). Choose $c_{0} \in(0, F(L) / 3)$. Since $u_{0}$ fulfils $(2.22)$, we get by integration over $[0, t]$

$$
\frac{u_{0}^{\prime 2}(t)}{2}+\int_{0}^{t} \frac{p^{\prime}(s)}{p(s)} u_{0}^{\prime 2}(s) \mathrm{d} s=F\left(B_{0}\right)-F\left(u_{0}(t)\right), \quad t>0
$$

For $t \rightarrow \infty$, we get, by (2.21),

$$
\int_{0}^{\infty} \frac{p^{\prime}(s)}{p(s)} u_{0}^{\prime 2}(s) \mathrm{d} s=F\left(B_{0}\right)
$$

Therefore, we can find $b>0$ such that

$$
\int_{b}^{\infty} \frac{p^{\prime}(s)}{p(s)} u_{0}^{\prime 2}(s) \mathrm{d} s<c_{0}
$$

Let $M_{b}$ be the constant of Lemma 2.1. Choose $\epsilon \in\left(0, c_{0} / 2 M_{b}\right)$. Assume that $B<0$ and $u$ is a corresponding solution of problem (2.13), (1.10). Using Lemma 2.1, Lemma 2.3 and the continuity of $F$, we can find $\delta>0$ such that if $\left|B-B_{0}\right|<\delta$, then

$$
\left|F(B)-F\left(B_{0}\right)\right|<c_{0},
$$


moreover $\left|u_{0}^{\prime}(t)-u^{\prime}(t)\right|<\epsilon$ for $t \in[0, b]$ and

$$
\begin{aligned}
\int_{0}^{b} \frac{p^{\prime}(s)}{p(s)}\left|u_{0}^{\prime 2}(s)-u^{\prime 2}(s)\right| \mathrm{d} s & \leq \max _{t \in[0, b]}\left|u_{0}^{\prime}(t)-u^{\prime}(t)\right| \int_{0}^{b} \frac{p^{\prime}(s)}{p(s)}\left(\left|u_{0}^{\prime}(s)\right|+\left|u^{\prime}(s)\right|\right) \mathrm{d} s \\
& \leq \epsilon \cdot 2 M_{b}<\frac{c_{0}}{2 M_{b}} 2 M_{b}=c_{0} .
\end{aligned}
$$

Therefore, we have

$$
\int_{0}^{b} \frac{p^{\prime}(s)}{p(s)}\left|u_{0}^{\prime 2}(s)-u^{\prime 2}(s)\right| \mathrm{d} s<c_{0} .
$$

Consequently, integrating (2.13) over $[0, t]$ and using (3.19)-(3.23), we get for $t \geq b$

$$
\begin{aligned}
F(B)-\tilde{F}(u(t))= & \int_{0}^{t} \frac{p^{\prime}(s)}{p(s)} u^{\prime 2}(s) \mathrm{d} s+\frac{u^{\prime 2}(t)}{2} \geq \int_{0}^{t} \frac{p^{\prime}(s)}{p(s)} u^{\prime 2}(s) \mathrm{d} s \\
\geq & \int_{0}^{b} \frac{p^{\prime}(s)}{p(s)} u^{\prime 2}(s) \mathrm{d} s=\int_{0}^{b} \frac{p^{\prime}(s)}{p(s)}\left(u^{\prime 2}(s)-u_{0}^{\prime 2}(s)\right) \mathrm{d} s \\
& +\int_{0}^{b} \frac{p^{\prime}(s)}{p(s)} u_{0}^{\prime 2}(s) \mathrm{d} s>-c_{0}+\int_{0}^{b} \frac{p^{\prime}(s)}{p(s)} u_{0}^{\prime 2}(s) \mathrm{d} s \\
= & -c_{0}+\int_{0}^{\infty} \frac{p^{\prime}(s)}{p(s)} u_{0}^{\prime 2}(s) \mathrm{d} s-\int_{b}^{\infty} \frac{p^{\prime}(s)}{p(s)} u_{0}^{\prime 2}(s) \mathrm{d} s \\
> & -c_{0}+F\left(B_{0}\right)-c_{0}=-2 c_{0}+F\left(B_{0}\right)-F(B)+F(B) \\
> & -3 c_{0}+F(B) .
\end{aligned}
$$

We get $\widetilde{F}(u(t))<3 c_{0}<F(L)$ for $t \geq b$. Therefore, $\widetilde{F}(u(t))=F(u(t))$ for $t \geq b$ and, due to (1.4)-(1.6),

$$
\sup \{u(t): t \in[b, \infty)\}<L
$$

Assume that there is $b_{0} \in(0, b)$ such that $u\left(b_{0}\right)=L, u^{\prime}\left(b_{0}\right)>0$. Then, since $\left(p(t) u^{\prime}(t)\right)^{\prime}=0$ if $t>b_{0}$ and $u(t)>L$, we get $u^{\prime}(t)>0$ and $u(t)>L$ for $t>b_{0}$, contrary to (3.25). Hence we get that $u$ fulfils (3.1).

\section{Escape Solutions}

During the whole section, we assume (1.3)-(1.8) and (1.13). We prove that problem (1.1), (1.10) has at least one escape solution. According to Section 1 and Remark 2.2, we work with the following definitions. 
Definition 4.1. Let $c>0$. A solution of problem (1.1), (1.10) on $[0, c]$ is called an escape solution if

$$
u(c)=L, \quad u^{\prime}(t)>0 \text { for } t \in(0, c] .
$$

Definition 4.2. A solution $u$ of problem (2.13), (1.10) is called an escape solution, if there exists $c>0$ such that

$$
u(c)=L, \quad u^{\prime}(t)>0 \quad \text { for } t \in(0, \infty) .
$$

Remark 4.3. If $u$ is an escape solution of problem (2.13), (1.10), then $u$ is an escape solution of problem (1.1), (1.10) on some interval $[0, c]$.

Theorem 4.4 (on three types of solutions.). Let $u$ be a solution of problem (1.1), (1.10). Then $u$ is just one of the following three types

(I) $u$ is damped;

(II) $u$ is homoclinic;

(III) $u$ is escape.

Proof. By Definition 3.1, $u$ is damped if and only if (3.1) holds. By Lemma 3.5 and Definition 1.3, $u$ is homoclinic if and only if (3.10) holds. Let $u$ be neither damped nor homoclinic. Then there exists $c>0$ such that $u$ is bounded on $[0, c], u(c)=L, u^{\prime}(c)>0$. So, $u$ has its first zero $\theta \in(0, c)$ and $u^{\prime}(t)>0$ on $(0, \theta]$. Assume that there exist $a_{1} \in(\theta, c)$ such that $u\left(a_{1}\right) \in(0, L)$ and $u^{\prime}\left(a_{1}\right)=0$. Then, by Lemma 2.6, either $u$ fulfils (2.21) or $u$ has its second zero and, arguing as in Steps $2-5$ of the proof of Theorem 3.3, we deduce that $u$ is a damped solution. This contradiction implies that $u^{\prime}(t)>0$ on $(0, c]$. Therefore, by Definition $4.1, u$ is an escape solution.

Theorem 4.5. Let $\mathcal{M}_{e} \subset(-\infty, 0)$ be the set of all B such that the corresponding solutions of (1.1), (1.10) are escape solutions. The set $\boldsymbol{M}_{e}$ is open in $(-\infty, 0)$.

Proof. Let $B_{0} \in \mathcal{M}_{e}$ and $u_{0}$ be a solution of problem (1.1), (1.10) with $B=B_{0}$. So, $u_{0}$ fulfils (4.1) for some $c>0$. Let $\tilde{u}_{0}$ be a solution of problem (2.13), (1.10) with $B=B_{0}$. Then $u_{0}=\tilde{u}_{0}$ on $[0, c]$ and $\tilde{u}_{0}$ is increasing on $[c, \infty)$. There exists $\varepsilon>0$ and $c_{0}>c$ such that $\tilde{u}_{0}\left(c_{0}\right)=L+\varepsilon$. Let $u_{1}$ be a solution of problem (2.13), (1.10) for some $B_{1}<0$. Lemma 2.3 yields $\delta>0$ such that if $\left|B_{1}-B_{0}\right|<\delta$, then $u_{1}\left(c_{0}\right)>\widetilde{u}_{0}\left(c_{0}\right)-\varepsilon=L$. Therefore, $u_{1}$ is an escape solution of problem (2.13), (1.10). By Remark 4.3, $u_{1}$ is also an escape solution of problem (1.1), (1.10) on some interval $\left[0, c_{1}\right] \subset\left[0, c_{0}\right]$. lemmas.

To prove that the set $\boldsymbol{M}_{e}$ of Theorem 4.5 is nonempty we will need the following two

Lemma 4.6. Let $B<0$. Assume that $u$ is a solution of problem (1.1), (1.10) on $[0, b)$ and $[0, b)$ is a maximal interval where $u$ is increasing and $u(t) \in[B, L]$ for $t \in[0, b)$. Then

$$
\int_{0}^{t} 2 F(u(s)) p(s) p^{\prime}(s) \mathrm{d} s=F(u(t)) p^{2}(t)+\frac{1}{2} p^{2}(t) u^{\prime 2}(t), \quad t \in(0, b) .
$$


Proof.

Step 1. We show that the interval $(0, b)$ is nonempty. Since $u(0)=B<0$ and $f$ satisfies (1.3), (1.13), we can find $\theta>0$ such that

$$
u(t)<0, \quad f(u(t))>0 \quad \text { for } t \in(0, \theta)
$$

Integrating (1.1) over $(0, t)$, we obtain

$$
u^{\prime}(t)=\frac{1}{p(t)} \int_{0}^{t} p(s) f(u(s)) \mathrm{d} s>0 \quad \text { for } t \in(0, \theta]
$$

So, $u$ is an increasing solution of problem (1.1), (1.10) on $[0, \theta]$ and $u(t) \in[B, 0]$ for $t \in[0, \theta]$. Therefore the nonempty interval $[0, b)$ exists.

Step 2. By multiplication of (1.1) by $p u^{\prime}$ and integration over $(0, t)$, we obtain

$$
\frac{1}{2} p^{2}(t) u^{\prime 2}(t)=\int_{0}^{t} f(u(s)) u^{\prime}(s) p^{2}(s) \mathrm{d} s, \quad t \in(0, b) .
$$

Using the "per partes" integration, we get for $t \in(0, b)$

$$
\int_{0}^{t} f(u(s)) u^{\prime}(s) p^{2}(s) \mathrm{d} s=-F(u(t)) p^{2}(t)+\int_{0}^{t} 2 F(u(s)) p(s) p^{\prime}(s) \mathrm{d} s .
$$

This relation together with (4.6) implies (4.3).

Remark 4.7. Consider a solution $u$ of Lemma 4.6. If $u$ is an escape solution, then $b<\infty$. Assume that $u$ is not an escape solution. Then both possibilities $b<\infty$ and $b=\infty$ can occur. Let $b<\infty$. By Theorem 4.4 and Lemma 2.5, $u(b) \in(0, L), u^{\prime}(b)=0$. Let $b=\infty$. We write $u(b)=\lim _{t \rightarrow \infty} u(t), u^{\prime}(b)=\lim _{t \rightarrow \infty} u^{\prime}(t)$. Using Lemmas 3.5 and 2.5 and Theorem 4.4, we obtain $u^{\prime}(b)=0$ and either $u(b)=0$ or $u(b)=L$.

Lemma 4.8. Let $C<\bar{B}$ and let $\left\{B_{n}\right\}_{n=1}^{\infty} \subset(-\infty, C)$. Then for each $n \in \mathbb{N}$ :

(i) there exists a solution $u_{n}$ of problem (1.1), (1.10) with $B=B_{n}$,

(ii) there exists $b_{n}>0$ such that $\left[0, b_{n}\right)$ is the maximal interval on which the solution $u_{n}$ is increasing and its values in this interval are contained in $\left[B_{n}, L\right]$,

(iii) there exists $\gamma_{n} \in\left(0, b_{n}\right)$ satisfying $u_{n}\left(\gamma_{n}\right)=C$.

If the sequence $\left\{\gamma_{n}\right\}_{n=1}^{\infty}$ is unbounded, then there exists $\ell \in \mathbb{N}$ such that $u_{\ell}$ is an escape solution. 
Proof. Similar arugmets can be found in [12]. By Lemma 2.1, the assertion (i) holds. The arguments in Step 1 of the proof of Lemma 4.6 imply (ii). The strict monotonicity of $u_{n}$ and Remark 4.7 yields a unique $\gamma_{n}$. Assume that $\left\{\gamma_{n}\right\}_{n=1}^{\infty}$ is unbounded. Then

$$
\lim _{n \rightarrow \infty} \gamma_{n}=\infty, \quad \gamma_{n}<b_{n}, n \in \mathbb{N}
$$

(otherwise, we take a subsequence). Assume on the contrary that for any $n \in \mathbb{N}, u_{n}$ is not an escape solution. Choose $n \in \mathbb{N}$. Then, by Remark 4.7,

$$
u_{n}\left(b_{n}\right) \in[0, L], \quad u_{n}^{\prime}\left(b_{n}\right)=0
$$

Due to (4.9), (1.2) and (ii) there exists $\bar{\gamma}_{n} \in\left[\gamma_{n}, b_{n}\right)$ satisfying

$$
u_{n}^{\prime}\left(\bar{\gamma}_{n}\right)=\max \left\{u_{n}^{\prime}(t): t \in\left[\gamma_{n}, b_{n}\right)\right\}
$$

By (i) and (ii), $u_{n}$ satisfies

$$
u_{n}^{\prime \prime}(t)+\frac{p^{\prime}(t)}{p(t)} u_{n}^{\prime}(t)=f\left(u_{n}(t)\right), \quad t \in\left(0, b_{n}\right)
$$

Integrating it over $[0, t]$, we get

$$
\frac{u_{n}^{\prime 2}(t)}{2}+F\left(u_{n}(t)\right)=F\left(B_{n}\right)-\int_{0}^{t} \frac{p^{\prime}(s)}{p(s)} u_{n}^{\prime 2}(s) \mathrm{d} s, \quad t \in\left(0, b_{n}\right)
$$

Put

$$
E_{n}(t)=\frac{u_{n}^{\prime 2}(t)}{2}+F\left(u_{n}(t)\right), \quad t \in\left(0, b_{n}\right)
$$

Then, by (4.12),

$$
\frac{\mathrm{d} E_{n}(t)}{\mathrm{d} t}=-\frac{p^{\prime}(t)}{p(t)} u_{n}^{\prime 2}(t)<0, \quad t \in\left(0, b_{n}\right)
$$

We see that $E_{n}$ is decreasing. From (1.4) and (1.6) we get that $F$ is increasing on $[0, L]$ and consequently by (4.9) and (4.13), we have

$$
E_{n}\left(\gamma_{n}\right)>F\left(u_{n}\left(\gamma_{n}\right)\right)=F(C), \quad E_{n}\left(b_{n}\right)=F\left(u_{n}\left(b_{n}\right)\right) \leq F(L) .
$$

Integrating (4.14) over $\left(\gamma_{n}, b_{n}\right)$ and using (4.10), we obtain

$$
E_{n}\left(\gamma_{n}\right)-E_{n}\left(b_{n}\right)=\int_{\gamma_{n}}^{b_{n}} \frac{p^{\prime}(t)}{p(t)} u_{n}^{\prime 2}(t) \mathrm{d} t \leq u_{n}^{\prime}\left(\bar{\gamma}_{n}\right)(L-C) K_{n},
$$


where

$$
K_{n}=\sup \left\{\frac{p^{\prime}(t)}{p(t)}: \quad t \in\left[\gamma_{n}, b_{n}\right)\right\} \in(0, \infty)
$$

Further, by (4.15),

$$
\begin{gathered}
F(C)<E_{n}\left(\gamma_{n}\right) \leq F(L)+u_{n}^{\prime}\left(\bar{\gamma}_{n}\right)(L-C) K_{n}, \\
\frac{F(C)-F(L)}{L-C} \cdot \frac{1}{K_{n}}<u_{n}^{\prime}\left(\bar{\gamma}_{n}\right) .
\end{gathered}
$$

Conditions (1.8) and (4.8) yield $\lim _{n \rightarrow \infty} K_{n}=0$, which implies

$$
\lim _{n \rightarrow \infty} u_{n}^{\prime}\left(\bar{\gamma}_{n}\right)=\infty
$$

By (4.13) and (4.18),

$$
\frac{u_{n}^{\prime 2}\left(\bar{\gamma}_{n}\right)}{2} \leq E_{n}\left(\bar{\gamma}_{n}\right) \leq E_{n}\left(\gamma_{n}\right) \leq F(L)+u_{n}^{\prime}\left(\bar{\gamma}_{n}\right)(L-C) K_{n}
$$

and consequently

$$
u_{n}^{\prime}\left(\bar{\gamma}_{n}\right)\left(\frac{1}{2} u_{n}^{\prime}\left(\bar{\gamma}_{n}\right)-(L-C) K_{n}\right) \leq F(L)<\infty, \quad n \in \mathbb{N}
$$

which contradicts (4.20). Therefore, at least one escape solution of (1.1), (1.10) with $B<\bar{B}$ must exist.

Theorem 4.9 (on escape solution.). Assume that (1.3)-(1.8) and (1.13) hold and let

$$
0<\liminf _{x \rightarrow-\infty} \frac{|x|}{f(x)}<\infty
$$

Then there exists $B<\bar{B}$ such that the corresponding solution of problem (1.1), (1.10) is an escape solution.

Proof. Let $C<\bar{B}$ and let $\left\{B_{n}\right\}_{n=1}^{\infty},\left\{u_{n}\right\}_{n=1}^{\infty},\left\{b_{n}\right\}_{n=1}^{\infty}$, and $\left\{\gamma_{n}\right\}_{n=1}^{\infty}$ be sequences from Lemma 4.8. Moreover, let

$$
\lim _{n \rightarrow \infty} B_{n}=-\infty
$$

By (4.24) we can find $n_{0} \in \mathbb{N}$ such that $B_{n}<2 C$ for $n \geq n_{0}$. We assume that for any $n \in \mathbb{N}, u_{n}$ is not an escape solution and we construct a contradiction. 
Step 1 . We derive some inequality for $u_{n}^{\prime}$. By Remark 4.7 , we have

$$
u_{n}\left(b_{n}\right) \in[0, L], \quad u_{n}^{\prime}\left(b_{n}\right)=0, \quad n \in \mathbb{N},
$$

and, by Lemma 4.8 , the sequence $\left\{\gamma_{n}\right\}_{n=1}^{\infty}$ is bounded. Therefore there exists $\Gamma \in(0, \infty)$ such that

$$
\gamma_{n} \leq \Gamma, \quad n \in \mathbb{N} .
$$

Choose an arbitrary $n \geq n_{0}$. According to Lemma 4.6, $u_{n}$ satisfies equality (4.3), that is

$$
\int_{0}^{t} 2 F\left(u_{n}(s)\right) p(s) p^{\prime}(s) \mathrm{d} s=F\left(u_{n}(t)\right) p^{2}(t)+\frac{1}{2} p^{2}(t) u_{n}^{\prime 2}(t), \quad t \in\left(0, b_{n}\right) .
$$

Since $u_{n}(0)=B_{n}<2 C<0$ and $u_{n}$ is increasing on $\left[0, b_{n}\right)$, there exists a unique $\bar{\gamma}_{n} \in\left(0, \gamma_{n}\right)$ such that

$$
u_{n}\left(\bar{\gamma}_{n}\right)=\frac{1}{2} B_{n}<C=u_{n}\left(\gamma_{n}\right)
$$

Having in mind, due to (1.4)-(1.8), that the inequality

$$
F\left(u_{n}(t)\right) p(t) p^{\prime}(t) \geq 0 \quad \text { for } t \in\left[0, b_{n}\right)
$$

holds, we get

$$
\int_{0}^{t} 2 F\left(u_{n}(s)\right) p(s) p^{\prime}(s) \mathrm{d} s>\int_{0}^{\bar{\gamma}_{n}} 2 F\left(u_{n}(s)\right) p(s) p^{\prime}(s) \mathrm{d} s, \quad t \in\left[\gamma_{n}, b_{n}\right)
$$

By virtue of (1.6) and (1.13), we see that $F$ is decreasing on $(-\infty, 0)$, which yields

$$
\min \left\{F\left(u_{n}(t)\right): t \in\left[0, \bar{\gamma}_{n}\right]\right\}=F\left(u_{n}\left(\bar{\gamma}_{n}\right)\right)=F\left(\frac{B_{n}}{2}\right)
$$

Hence,

$$
\int_{0}^{t} 2 F\left(u_{n}(s)\right) p(s) p^{\prime}(s) \mathrm{d} s>F\left(\frac{B_{n}}{2}\right) p^{2}\left(\bar{\gamma}_{n}\right), \quad t \in\left[\gamma_{n}, b_{n}\right)
$$

Since $u_{n}\left(\gamma_{n}\right)=C$ and $u_{n}\left(b_{n}\right) \in[0, L]$, the monotonicity of $u_{n}$ yields $u_{n}(t) \in[C, L]$ for $t \in$ $\left[\gamma_{n}, b_{n}\right]$, and consequently

$$
\max \left\{F\left(u_{n}(t)\right): t \in\left[\gamma_{n}, b_{n}\right)\right\}=F(C)
$$


Therefore (4.27) and (4.32) give

$$
F\left(\frac{B_{n}}{2}\right) \frac{p^{2}\left(\bar{\gamma}_{n}\right)}{p^{2}(t)}<F(C)+\frac{1}{2} u_{n}^{\prime 2}(t), \quad t \in\left[\gamma_{n}, b_{n}\right) .
$$

Step 2. We prove that the sequence $\left\{\bar{\gamma}_{n}\right\}_{n=1}^{\infty}$ is bounded below by some positive number. Since $u_{n}$ is a solution of $(1.1)$ on $\left[0, b_{n}\right)$, we have

$$
\left(p(t) u_{n}^{\prime}(t)\right)^{\prime}=p(t) f\left(u_{n}(t)\right), \quad t \in\left(0, \bar{\gamma}_{n}\right)
$$

Integrating it, we get

$$
u_{n}^{\prime}(t)=\frac{1}{p(t)} \int_{0}^{t} p(s) f\left(u_{n}(s)\right) \mathrm{d} s \leq f\left(\sigma_{n} B_{n}\right) \frac{P(t)}{p(t)}, \quad t \in\left(0, \bar{\gamma}_{n}\right)
$$

where $\sigma_{n} \in[1 / 2,1]$ satisfies $f\left(\sigma_{n} B_{n}\right)=\max \left\{f(x): x \in\left[B_{n},(1 / 2) B_{n}\right]\right\}$ and $P(t)=\int_{0}^{t} p(s) \mathrm{d}$. Having in mind (1.8), we see that $p$ is increasing and $0<P(t) / p(t) \leq t$ for $t \in(0, \infty)$. Consequently

$$
\lim _{t \rightarrow 0+} \int_{0}^{t} \frac{P(s)}{p(s)} \mathrm{d} s=0
$$

Integrating (4.36) over $\left(0, \bar{\gamma}_{n}\right)$, we obtain

$$
\frac{1}{2} B_{n}-B_{n} \leq f\left(\sigma_{n} B_{n}\right) \int_{0}^{\bar{\gamma}_{n}} \frac{P(s)}{p(s)} \mathrm{d} s,
$$

and hence

$$
\int_{0}^{\bar{\gamma}_{n}} \frac{P(s)}{p(s)} \mathrm{d} s \geq \frac{1}{2} \frac{\left|B_{n}\right|}{f\left(\sigma_{n} B_{n}\right)}
$$

By (4.23) we get

$$
\liminf _{n \rightarrow \infty} \frac{1}{2} \frac{\left|B_{n}\right|}{f\left(\sigma_{n} B_{n}\right)}=\liminf _{n \rightarrow \infty} \frac{1}{2 \sigma_{n}} \frac{\left|\sigma_{n} B_{n}\right|}{f\left(\sigma_{n} B_{n}\right)}>0,
$$

which, due to (4.39), yields

$$
\liminf _{n \rightarrow \infty} \int_{0}^{\bar{r}_{n}} \frac{P(s)}{p(s)} \mathrm{d} s>0
$$

So, by virtue of (4.37), there exists $\gamma_{0}>0$ such that $\bar{\gamma}_{n} \geq \gamma_{0}$ for $n \geq n_{0}$. 
Step 3. We construct a contradiction. Putting $\gamma_{0}$ in (4.34), we have

$$
F\left(\frac{B_{n}}{2}\right) \frac{p^{2}\left(\gamma_{0}\right)}{p^{2}(t)}-F(C)<\frac{1}{2} u_{n}^{\prime 2}(t), \quad t \in\left[\gamma_{n}, b_{n}\right)
$$

Due to (4.23), $\lim _{x \rightarrow-\infty} f(x)=\infty$. Therefore, $\lim _{x \rightarrow-\infty} F(x)=\infty$, and consequently, by (4.24),

$$
\lim _{n \rightarrow \infty} F\left(\frac{B_{n}}{2}\right)=\infty
$$

In order to get a contradiction, we distinguish two cases.

Case 1. Let $\lim \sup _{n \rightarrow \infty} b_{n}<\infty$, that is, we can find $b_{0}>0, n_{1} \in \mathbb{N}, n_{1} \geq n_{0}$, such that

$$
b_{n} \leq b_{0} \quad \text { for } n \in \mathbb{N}, n \geq n_{1}
$$

Then, by (4.43), for each sufficiently large $n \in \mathbb{N}$, we get

$$
F\left(\frac{B_{n}}{2}\right)>\frac{p^{2}\left(b_{0}\right)}{p^{2}\left(\gamma_{0}\right)}\left(F(C)+\frac{1}{2}\right)
$$

Putting it to (4.42), we have

$$
\frac{1}{2}<F\left(\frac{B_{n}}{2}\right) \frac{p^{2}\left(\gamma_{0}\right)}{p^{2}\left(b_{0}\right)}-F(C)<\frac{1}{2} u_{n}^{\prime 2}(t), \quad t \in\left[\gamma_{n}, b_{n}\right) .
$$

Therefore $1 \leq u_{n}^{\prime}\left(b_{n}\right)$, contrary to (4.25).

Case 2. Let $\limsup _{n \rightarrow \infty} b_{n}=\infty$. We may assume $\lim _{n \rightarrow \infty} b_{n}=\infty$ (otherwise we take a subsequence). Then there exists $n_{2} \in \mathbb{N}, n_{2} \geq n_{0}$, such that

$$
\Gamma+1 \leq b_{n} \text { for } n \in \mathbb{N}, n \geq n_{2} .
$$

Due to (4.43), for each sufficiently large $n \in \mathbb{N}$, we get

$$
F\left(\frac{B_{n}}{2}\right)>\frac{p^{2}(\Gamma+1)}{p^{2}\left(\gamma_{0}\right)}\left(F(C)+\frac{1}{2}(L-C)^{2}\right)
$$

Putting it to (4.42), we have

$$
\frac{1}{2}(L-C)^{2}<F\left(\frac{B_{n}}{2}\right) \frac{p^{2}\left(\gamma_{0}\right)}{p^{2}(\Gamma+1)}-F(C)<\frac{1}{2} u_{n}^{\prime 2}(t), \quad t \in\left[\gamma_{n}, \Gamma+1\right]
$$


Therefore, $L-C<u_{n}^{\prime}(t)$ for $t \in\left[\gamma_{n}, \Gamma+1\right]$. Integrating it over $\left[\gamma_{n}, \Gamma+1\right]$, we obtain

$$
(L-C)\left(\Gamma+1-\gamma_{n}\right)<u_{n}(\Gamma+1)-u_{n}\left(\gamma_{n}\right)=u_{n}(\Gamma+1)-C,
$$

which yields, by (4.26), $L<u_{n}(\Gamma+1)$ and also $L<u_{n}\left(b_{n}\right)$, contrary to (4.25). These contradictions obtained in both cases imply that there exists $\ell \in \mathbb{N}$ such that $u_{\ell}$ is an escape solution.

\section{Homoclinic Solution}

The following theorem provides the existence of a homoclinic solution under the assumption that the function $f$ in (1.1) has a linear behaviour near $-\infty$. According to Definition 1.2, a homoclinic solution is a strictly increasing solution of problem (1.1), (1.2).

Theorem 5.1 (on homoclinic solution). Let the assumptions of Theorem 4.9 be satisfied. Then there exists $B<\bar{B}$ such that the corresponding solution of problem (1.1), (1.10) is a homoclinic solution.

Proof. For $B<0$ denote by $u_{B}$ the corresponding solution of problem (1.1), (1.10). Let $\boldsymbol{M}_{d}$ and $\boldsymbol{M}_{e}$ be the set of all $B<0$ such that $u_{B}$ is a damped solution and an escape solution, respectively. By Theorems 3.7, 3.8, 4.5, and 4.9, the sets $\mathcal{M}_{d}$ and $\boldsymbol{M}_{e}$ are nonempty and open in $(-\infty, 0)$. Therefore, the set $\mathcal{M}_{h}=(-\infty, 0) \backslash\left(\mathcal{M}_{d} \cup \mathcal{M}_{e}\right)$ is nonempty. Choose $B^{*} \in \mathcal{M}_{h}$. Then, by Theorem $4.4, u_{B^{*}}$ is a homoclinic solution. Moreover, due to Theorem 3.7, $B^{*}<\bar{B}$.

Example 5.2. The function

$$
f(x)= \begin{cases}c_{0} x & \text { for } x<0, \\ x(x-L) & \text { for } x \in[0, L]\end{cases}
$$

where $c_{0}$ is a negative constant, satisfies the conditions (1.3)-(1.6), (1.13), and (4.23).

\section{Acknowledgments}

The authors thank the referee for valuable comments. This work was supported by the Council of Czech Government MSM 6198959214.

\section{References}

[1] F. Dell'Isola, H. Gouin, and G. Rotoli, “Nucleation of spherical shell-like interfaces by second gradient theory: numerical simulations," European Journal of Mechanics. B, vol. 15, no. 4, pp. 545-568, 1996.

[2] G. H. Derrick, "Comments on nonlinear wave equations as models for elementary particles," Journal of Mathematical Physics, vol. 5, pp. 1252-1254, 1964.

[3] H. Gouin and G. Rotoli, "An analytical approximation of density profile and surface tension of microscopic bubbles for Van Der Waals fluids," Mechanics Research Communications, vol. 24, no. 3, pp. 255-260, 1997.

[4] G. Kitzhofer, O. Koch, P. Lima, and E. Weinmüller, "Efficient numerical solution of the density profile equation in hydrodynamics," Journal of Scientific Computing, vol. 32, no. 3, pp. 411-424, 2007. 
[5] P. M. Lima, N. B. Konyukhova, A. I. Sukov, and N. V. Chemetov, "Analytical-numerical investigation of bubble-type solutions of nonlinear singular problems," Journal of Computational and Applied Mathematics, vol. 189, no. 1-2, pp. 260-273, 2006.

[6] O. Koch, P. Kofler, and E. B. Weinmüller, "Initial value problems for systems of ordinary first and second order differential equations with a singularity of the first kind," Analysis, vol. 21, no. 4, pp. 373-389, 2001.

[7] D. Bonheure, J. M. Gomes, and L. Sanchez, "Positive solutions of a second-order singular ordinary differential equation," Nonlinear Analysis: Theory, Methods E Applications, vol. 61, no. 8, pp. 1383-1399, 2005.

[8] M. Conti, L. Merizzi, and S. Terracini, "Radial solutions of superlinear equations on $\mathbb{R}^{N}$. I. A global variational approach," Archive for Rational Mechanics and Analysis, vol. 153, no. 4, pp. 291-316, 2000.

[9] H. Berestycki, P.-L. Lions, and L. A. Peletier, "An ODE approach to the existence of positive solutions for semilinear problems in $\mathbb{R}^{N}$," Indiana University Mathematics Journal, vol. 30, no. 1, pp. 141-157, 1981.

[10] L. Maatoug, "On the existence of positive solutions of a singular nonlinear eigenvalue problem," Journal of Mathematical Analysis and Applications, vol. 261, no. 1, pp. 192-204, 2001.

[11] I. Rachůnková and J. Tomeček, "Singular nonlinear problem for ordinary differential equation of the second-order on the half-line," in Mathematical Models in Engineering, Biology and Medicine: International Conference on Boundary Value Problems, A. Cabada, E. Liz, and J. J. Nieto, Eds., pp. 294-303, 2009.

[12] I. Rachůnková and J. Tomeček, "Bubble-type solutions of nonlinear singular problem," submitted.

[13] A. P. Palamides and T. G. Yannopoulos, "Terminal value problem for singular ordinary differential equations: theoretical analysis and numerical simulations of ground states," Boundary Value Problems, vol. 2006, Article ID 28719, 28 pages, 2006. 